\title{
TIKHONOV REGULARIZATION FOR DUNKL MULTIPLIER OPERATORS
}

\author{
Fethi SOlTani*
}

\begin{abstract}
We study some class of Dunkl multiplier operators $T_{k, m}$; and we give for them an application of the theory of reproducing kernels to the Tikhonov regularization, which gives the best approximation of the operators $T_{k, m}$ on the Dunkl-type Paley-Wiener spaces $H_{h}$.
\end{abstract}

\section{Introduction}

In this paper, we consider $\mathbf{R}^{d}$ with the Euclidean inner product $\langle.,$.$\rangle and$ norm $|y|:=\sqrt{\langle y, y\rangle}$. For $\alpha \in \mathbf{R}^{d} \backslash\{0\}$, let $\sigma_{\alpha}$ be the reflection in the hyperplane $H_{\alpha} \subset \mathbf{R}^{d}$ orthogonal to $\alpha$ :

$$
\sigma_{\alpha} x:=x-\frac{2\langle\alpha, x\rangle}{|\alpha|^{2}} \alpha .
$$

A finite set $\Re \subset \mathbf{R}^{d} \backslash\{0\}$ is called a root system, if $\Re \cap \mathbf{R} . \alpha=\{-\alpha, \alpha\}$ and $\sigma_{\alpha} \Re=\Re$ for all $\alpha \in \Re$. We assume that it is normalized by $|\alpha|^{2}=2$ for all $\alpha \in \Re$. For a root system $\Re$, the reflections $\sigma_{\alpha}, \alpha \in \Re$, generate a finite group $G$. The Coxeter group $G$ is a subgroup of the orthogonal group $O(d)$. All reflections in $G$, correspond to suitable pairs of roots. For a given $\beta \in$ $\mathbf{R}^{d} \backslash \bigcup_{\alpha \in \Re} H_{\alpha}$, we fix the positive subsystem $\Re_{+}:=\{\alpha \in \Re:\langle\alpha, \beta\rangle>0\}$. Then for each $\alpha \in \Re$ either $\alpha \in \Re_{+}$or $-\alpha \in \Re_{+}$.

Let $k: \Re \rightarrow \mathbf{C}$ be a multiplicity function on $\Re$ (a function which are constant on the orbits under the action of $G$ ). As an abbreviation, we introduce the index $\gamma_{k}:=\sum_{\alpha \in \Re_{+}} k(\alpha)$.

Throughout this paper, we will assume that $k(\alpha) \geq 0$ for all $\alpha \in \Re$. Moreover, let $w_{k}$ denote the weight function $w_{k}(x):=\prod_{\alpha \in \Re_{+}}|\langle\alpha, x\rangle|^{2 k(\alpha)}$, for all $x \in \mathbf{R}^{d}$, which is $G$-invariant and homogeneous of degree $2 \gamma_{k}$.

2010 Mathematics Subject Classification. 42B10; 42B15; 46E35.

Key words and phrases. Dunkl multiplier operators, Dunkl-type Paley-Wiener space, Tikhonov regularization.

* Author partially supported by the DGRST research project LR11ES11 and CMCU program $10 \mathrm{G} / 1503$.

Received February 10, 2015. 
Let $c_{k}$ be the Mehta-type constant given by

$$
c_{k}:=\left(\int_{\mathbf{R}^{d}} e^{-|x|^{2} / 2} w_{k}(x) \mathrm{d} x\right)^{-1} .
$$

We denote by $\mu_{k}$ the measure on $\mathbf{R}^{d}$ given by $\mathrm{d} \mu_{k}(x):=c_{k} w_{k}(x) \mathrm{d} x$; and by $L^{p}\left(\mu_{k}\right), 1 \leq p \leq \infty$, the space of measurable functions $f$ on $\mathbf{R}^{d}$, such that

$$
\begin{aligned}
& \|f\|_{L^{p}\left(\mu_{k}\right)}:=\left(\int_{\mathbf{R}^{d}}|f(x)|^{p} \mathrm{~d} \mu_{k}(x)\right)^{1 / p}<\infty, \quad 1 \leq p<\infty, \\
& \|f\|_{L^{\infty}\left(\mu_{k}\right)}:=\operatorname{ess} \sup _{x \in \mathbf{R}^{d}}|f(x)|<\infty .
\end{aligned}
$$

For $f \in L^{1}\left(\mu_{k}\right)$ the Dunkl transform is defined (see [3]) by

$$
\mathscr{F}_{k}(f)(y):=\int_{\mathbf{R}^{d}} E_{k}(-i x, y) f(x) \mathrm{d} \mu_{k}(x), \quad y \in \mathbf{R}^{d},
$$

where $E_{k}(-i x, y)$ denotes the Dunkl kernel (for more details, see the next section).

Let $m$ be a function in $L^{\infty}\left(\mu_{k}\right)$. The Dunkl multiplier operators $T_{k, m}$, are defined for $f \in L^{2}\left(\mu_{k}\right)$ by

$$
T_{k, m} f(x):=\mathscr{\mathscr { F }}_{k}^{-1}\left(m \mathscr{F}_{k}(f)\right)(x), \quad x \in \mathbf{R}^{d} .
$$

These operators are studied in $[15,16]$ where the author established some applications (Calderón's reproducing formulas, best approximation formulas, extremal functions....).

Building on the ideas of Matsuura et al. [6], Saitoh [11, 13] and Yamada et al. [19], and using the theory of reproducing kernels [1, 10], we give best approximation of the operator $T_{k, m}$ on the Dunkl-type Paley-Wiener space $H_{h}$. More precisely, for all $\eta>0, g \in L^{2}\left(\mu_{k}\right)$, the infimum

$$
\inf _{f \in H_{h}}\left\{\eta\|f\|_{H_{h}}^{2}+\left\|g-T_{k, m} f\right\|_{L^{2}\left(\mu_{k}\right)}^{2}\right\}
$$

is attained at one function $F_{\eta, g}^{*}$, called the extremal function, and given by

$$
F_{\eta, g}^{*}(y)=\int_{\mathbf{R}^{d}} E_{k}(i y, z) \frac{\chi_{h}(z) \overline{m(z)} \mathscr{F}_{k}(g)(z)}{\eta+|m(z)|^{2}} \mathrm{~d} \mu_{k}(z) .
$$

Next we show for $F_{\eta, g}^{*}$ the following properties.

(i) $\left\|F_{\eta, g}^{*}\right\|_{H_{h}} \leq \frac{1}{2 \sqrt{\eta}}\|g\|_{L^{2}\left(\mu_{k}\right)}$.

(ii) $\lim _{\eta \rightarrow 0^{+}}\left\|T_{k, m} F_{\eta, g}^{*}-g\right\|_{L^{2}\left(\mu_{k}\right)}=0$.

(iii) $\lim _{\eta \rightarrow 0^{+}}\left\|F_{\eta, T_{k, m} f}^{*}-f\right\|_{H_{h}} \stackrel{=}{=}$.

In the Dunkl setting, the extremal functions are studied in several directions $[14,15,16,17]$. 
This paper is organized as follows. In Section 2 we define and study the Dunkl multiplier operators $T_{k, m}$ on the Dunkl-type Paley-Wiener spaces $H_{h}$. The last section of this paper is devoted to give an application of the theory of reproducing kernels to the Tikhonov regularization, which gives the best approximation of the operators $T_{k, m}$ on $H_{h}$.

\section{The Dunkl-type Paley-Wiener spaces}

The Dunkl operators $\mathscr{D}_{j} ; j=1, \ldots, d$, on $\mathbf{R}^{d}$ associated with the finite reflection group $G$ and multiplicity function $k$ are given, for a function $f$ of class $C^{1}$ on $\mathbf{R}^{d}$, by

$$
\mathscr{D}_{j} f(x):=\frac{\partial}{\partial x_{j}} f(x)+\sum_{\alpha \in \Re_{+}} k(\alpha) \alpha_{j} \frac{f(x)-f\left(\sigma_{\alpha} x\right)}{\langle\alpha, x\rangle} .
$$

For $y \in \mathbf{R}^{d}$, the initial problem $\mathscr{D}_{j} u(., y)(x)=y_{j} u(x, y), j=1, \ldots, d$, with $u(0, y)=1$ admits a unique analytic solution on $\mathbf{R}^{d}$, which will be denoted by $E_{k}(x, y)$ and called Dunkl kernel [2, 4]. This kernel has a unique analytic extension to $\mathbf{C}^{d} \times \mathbf{C}^{d}$ (see [8]). In our case (see [2, 3]),

$$
\left|E_{k}(i x, y)\right| \leq 1, \quad x, y \in \mathbf{R}^{d} .
$$

The Dunkl kernel gives rise to an integral transform, which is called Dunkl transform on $\mathbf{R}^{d}$, and was introduced by Dunkl in [3], where already many basic properties were established. Dunkl's results were completed and extended later by De Jeu [4]. The Dunkl transform of a function $f$ in $L^{1}\left(\mu_{k}\right)$, is defined by

$$
\mathscr{F}_{k}(f)(y):=\int_{\mathbf{R}^{d}} E_{k}(-i x, y) f(x) \mathrm{d} \mu_{k}(x), \quad y \in \mathbf{R}^{d} .
$$

We notice that $\mathscr{F}_{0}$ agrees with the Fourier transform $\mathscr{F}$ that is given by

$$
\mathscr{F}(f)(y):=(2 \pi)^{-d / 2} \int_{\mathbf{R}^{d}} e^{-i\langle x, y\rangle} f(x) \mathrm{d} x, \quad x \in \mathbf{R}^{d} .
$$
$[3,4])$.

Some of the properties of Dunkl transform $\mathscr{F}_{k}$ are collected bellow (see

THEOREM 2.1. (i) $L^{1}-L^{\infty}$-boundedness. For all $f \in L^{1}\left(\mu_{k}\right), \quad \mathscr{F}_{k}(f) \in$ $L^{\infty}\left(\mu_{k}\right)$ and

$$
\left\|\widetilde{F}_{k}(f)\right\|_{L^{\infty}\left(\mu_{k}\right)} \leq\|f\|_{L^{1}\left(\mu_{k}\right)} .
$$

(ii) Inversion theorem. Let $f \in L^{1}\left(\mu_{k}\right)$, such that $\mathscr{F}_{k}(f) \in L^{1}\left(\mu_{k}\right)$. Then

$$
f(x)=\mathscr{F}_{k}\left(\mathscr{F}_{k}(f)\right)(-x) \text {, a.e. } x \in \mathbf{R}^{d} .
$$


(iii) Plancherel theorem. The Dunkl transform $\mathscr{F}_{k}$ extends uniquely to an isometric isomorphism of $L^{2}\left(\mu_{k}\right)$ onto itself. In particular,

$$
\left\|\mathscr{F}_{k}(f)\right\|_{L^{2}\left(\mu_{k}\right)}=\|f\|_{L^{2}\left(\mu_{k}\right)} .
$$

Let $h>0$ and $\chi_{h}$ the function defined by

$$
\chi_{h}(z):=\prod_{i=1}^{d} \chi_{(-1 / h, 1 / h)}\left(z_{i}\right), \quad z=\left(z_{1}, \ldots, z_{d}\right) \in \mathbf{R}^{d},
$$

where $\chi_{(-1 / h, 1 / h)}$ is the characteristic function of the interval $(-1 / h, 1 / h)$.

We define the Paley-Wiener space $H_{h}$, as

$$
H_{h}:=\mathscr{F}_{k}^{-1}\left(\chi_{h} L^{2}\left(\mu_{k}\right)\right) \text {. }
$$

The space $H_{h}$ satisfies

$$
H_{h} \subset L^{2}\left(\mu_{k}\right), \quad \mathscr{F}_{k}\left(H_{h}\right) \subset L^{1} \cap L^{2}\left(\mu_{k}\right) .
$$

We see that any element $f \in H_{h}$ is represented uniquely by a function $F \in L^{2}\left(\mu_{k}\right)$ in the form

$$
f=\mathscr{F}_{k}^{-1}\left(\chi_{h} F\right) .
$$

The space $H_{h}$ provided with the norm

$$
\|f\|_{H_{h}}=\|F\|_{L^{2}\left(\mu_{k}\right)} \text {. }
$$

Let $m$ be a function in $L^{\infty}\left(\mu_{k}\right)$. The Dunkl multiplier operators $T_{k, m}$, are defined for $f \in L^{2}\left(\mu_{k}\right)$ by

$$
T_{k, m} f:=\mathscr{F}_{k}^{-1}\left(m \mathscr{F}_{k}(f)\right) .
$$

THEOREM 2.2. Let $m \in L^{\infty}\left(\mu_{k}\right)$. The operators $T_{k, m}$ are bounded linear operators from $H_{h}$ into $L^{2}\left(\mu_{k}\right)$, and

$$
\left\|T_{k, m} f\right\|_{L^{2}\left(\mu_{k}\right)} \leq\|m\|_{L^{\infty}\left(\mu_{k}\right)}\|f\|_{H_{h}} .
$$

Proof. Let $m \in L^{\infty}\left(\mu_{k}\right)$. From Theorem 2.1 (iii) and (2.3) we obtain

$$
\begin{aligned}
\left\|T_{k, m} f\right\|_{L^{2}\left(\mu_{k}\right)} & =\left(\int_{\mathbf{R}^{d}}|m(z)|^{2}\left|\mathscr{F}_{k}(f)(z)\right|^{2} \mathrm{~d} \mu_{k}(z)\right)^{1 / 2} \\
& \leq\|m\|_{L^{\infty}\left(\mu_{k}\right)}\left(\int_{\mathbf{R}^{d}} \chi_{h}(z)|F(z)|^{2} \mathrm{~d} \mu_{k}(z)\right)^{1 / 2} \\
& \leq\|m\|_{L^{\infty}\left(\mu_{k}\right)}\|f\|_{H_{h}} .
\end{aligned}
$$

This gives the result.

As application, we give the following example. 
Example 2.3. Let $m$ be the function defined for $t>0$ by

$$
m(z):=e^{-t|z|^{2}}, \quad z \in \mathbf{R}^{d} .
$$

Then $T_{k, m} f=W_{k, t}(f)$, where $W_{k, t}$ is the Dunkl-type Weierstrass transform $[9,14]$. $H_{h}$ by

Let $\eta>0$. We denote by $\langle., .\rangle_{\eta, H_{h}}$ the inner product defined on the space

$$
\langle f, g\rangle_{\eta, H_{h}}:=\eta\langle f, g\rangle_{H_{h}}+\left\langle T_{k, m} f, T_{k, m} g\right\rangle_{L^{2}\left(\mu_{k}\right)},
$$

and the norm $\|f\|_{\eta, H_{h}}:=\sqrt{\langle f, f\rangle_{\eta, H_{h}}}$.

On $H_{h}$ the two norms $\|\cdot\|_{H_{h}}$ and $\|\cdot\|_{\eta, H_{h}}$ are equivalent. This $\left(H_{h},\langle., .\rangle_{\eta, H_{h}}\right)$ is a Hilbert space with reproducing kernel given by the following theorem.

THEOREM 2.4. Let $\eta>0$ and $m \in L^{\infty}\left(\mu_{k}\right)$. The space $\left(H_{h},\langle., .\rangle_{\eta, H_{h}}\right)$ has the reproducing kernel

$$
K_{h}(x, y)=\int_{\mathbf{R}^{d}} \frac{\chi_{h}(z) E_{k}(i x, z) E_{k}(-i y, z)}{\eta+|m(z)|^{2}} \mathrm{~d} \mu_{k}(z),
$$

that is

(i) For all $y \in \mathbf{R}^{d}$, the function $x \rightarrow K_{h}(x, y)$ belongs to $H_{h}$.

(ii) The reproducing property: for all $f \in H_{h}$ and $y \in \mathbf{R}^{d}$,

$$
\left\langle f, K_{h}(., y)\right\rangle_{\eta, H_{h}}=f(y) .
$$

Proof. (i) Let $y \in \mathbf{R}^{d}$. From (2.1), the function $z \rightarrow \frac{\chi_{h}(z) E_{k}(-i y, z)}{\eta+|m(z)|^{2}}$ belongs to $L^{1} \cap L^{2}\left(\mu_{k}\right)$. Then, the function $K_{h}$ is well defined and by Theorem 2.1 (ii), we have

$$
K_{h}(x, y)=\mathscr{F}_{k}^{-1}\left(\frac{\chi_{h}(z) E_{k}(-i y, z)}{\eta+|m(z)|^{2}}\right)(x), \quad x \in \mathbf{R}^{d} .
$$

Then by Theorem 2.1 (iii) and (2.1), we obtain

$$
\left|\mathscr{F}_{k}\left(K_{h}(., y)\right)(z)\right| \leq \frac{\chi_{h}(z)}{\eta} \quad \text { and }\left\|K_{h}(., y)\right\|_{H_{h}}^{2} \leq \frac{c_{k} 2^{\gamma_{k}+d} d^{\gamma_{k}}}{\eta^{2} h^{2 \gamma_{k}+d}} .
$$

This proves that for all $y \in \mathbf{R}^{d}$ the function $K_{h}(., y)$ belongs to $H_{h}$.

(ii) Let $f \in H_{h}$ and $y \in \mathbf{R}^{d}$. From (2.4) and (2.6), we have

$$
\left\langle f, K_{h}(., y)\right\rangle_{\eta, H_{h}}=\int_{\mathbf{R}^{d}} E_{k}(i y, z) \mathscr{F}_{k}(f)(z) \mathrm{d} \mu_{k}(z),
$$

and from (2.2), we obtain the reproducing property:

$$
\left\langle f, K_{h}(., y)\right\rangle_{\eta, H_{h}}=f(y) .
$$

This completes the proof of the theorem. 


\section{Extremal functions for the operators $T_{k, m}$}

In this section, by using the theory of extremal function and reproducing kernel of Hilbert space $[10,11,12,13]$ we study the extremal function associated to the Dunkl multiplier operators $T_{k, m}$. In the particular case when $k=0$ this function is studied in $[7,18]$. The main result of this section can be stated as follows.

THEOREM 3.1. Let $m \in L^{\infty}\left(\mu_{k}\right)$. For any $g \in L^{2}\left(\mu_{k}\right)$ and for any $\eta>0$, there exists a unique function $F_{\eta, g}^{*}$, where the infimum

$$
\inf _{f \in H_{h}}\left\{\eta\|f\|_{H_{h}}^{2}+\left\|g-T_{k, m} f\right\|_{L^{2}\left(\mu_{k}\right)}^{2}\right\}
$$

is attained. Moreover, the extremal function $F_{\eta, g}^{*}$ is given by

$$
F_{\eta, g}^{*}(y)=\int_{\mathbf{R}^{d}} g(x) Q_{h}(x, y) \mathrm{d} \mu_{k}(x)
$$

where

$$
Q_{h}(x, y)=\int_{\mathbf{R}^{d}} \frac{\chi_{h}(z) \overline{m(z)} E_{k}(-i x, z) E_{k}(i y, z)}{\eta+|m(z)|^{2}} \mathrm{~d} \mu_{k}(z) .
$$

Proof. The existence and unicity of the extremal function $F_{\eta, g}^{*}$ satisfying (3.1) is obtained in $[5,6,12]$. Especially, $F_{\eta, g}^{*}$ is given by the reproducing kernel of $H_{h}$ with $\|\cdot\|_{\eta, H_{h}}$ norm as

$$
F_{\eta, g}^{*}(y)=\left\langle g, T_{k, m}\left(K_{h}(., y)\right)\right\rangle_{L^{2}\left(\mu_{k}\right)},
$$

where $K_{h}$ is the kernel given by (2.5).

But by Theorem 2.1 (ii) and (2.6), we have

$$
\begin{aligned}
T_{k, m}\left(K_{h}(., y)\right)(x) & =\int_{\mathbf{R}^{d}} m(z) \mathscr{F}_{k}\left(K_{h}(., y)\right)(z) E_{k}(i x, z) \mathrm{d} \mu_{k}(z) \\
& =\int_{\mathbf{R}^{d}} \frac{\chi_{h}(z) m(z) E_{k}(i x, z) E_{k}(-i y, z)}{\eta+|m(z)|^{2}} \mathrm{~d} \mu_{k}(z) .
\end{aligned}
$$

This clearly yields the result.

As application, we give the following example.

Example 3.2. Let $\eta>0$ and $g \in L^{2}\left(\mu_{k}\right)$. If $m(z):=e^{-t|z|^{2}}, t>0$, then

$$
F_{\eta, g}^{*}(y)=\int_{\mathbf{R}^{d}} g(x) Q_{h}(x, y) \mathrm{d} \mu_{k}(x),
$$

where

$$
Q_{h}(x, y)=\int_{\mathbf{R}^{d}} \frac{\chi_{h}(z) E_{k}(-i x, z) E_{k}(i y, z)}{\eta e^{t|z|^{2}}+e^{-t|z|^{2}}} \mathrm{~d} \mu_{k}(z) .
$$


COROLlary 3.3. Let $\eta>0$ and $g \in L^{2}\left(\mu_{k}\right)$. The extremal function $F_{\eta, g}^{*}$ satisfies:

(i) $\left|F_{\eta, g}^{*}(y)\right| \leq\left(\frac{c_{k} 2^{\gamma_{k}+d} d^{\gamma_{k}}}{4 \eta h^{2 \gamma_{k}+d}}\right)^{1 / 2}\|g\|_{L^{2}\left(\mu_{k}\right)}$.
(ii) $\left\|F_{\eta, g}^{*}\right\|_{L^{2}\left(\mu_{k}\right)} \leq\left(\frac{c_{k} 2^{\gamma_{k}+d} d^{\gamma_{k}}}{4 \eta h^{2 \gamma_{k}+d}}\right)^{1 / 2}\left(\int_{\mathbf{R}^{d}}|g(x)|^{2} e^{|x|^{2} / 2} \mathrm{~d} \mu_{k}(x)\right)^{1 / 2}$.

Proof. (i) From (3.2) and Theorem 2.1 (iii), we have

$$
\begin{aligned}
\left|F_{\eta, g}^{*}(y)\right| & \leq\|g\|_{L^{2}\left(\mu_{k}\right)}\left\|T_{k, m}\left(K_{h}(., y)\right)\right\|_{L^{2}\left(\mu_{k}\right)} \\
& \leq\|g\|_{L^{2}\left(\mu_{k}\right)}\left\|m \mathscr{F}_{k}\left(K_{h}(., y)\right)\right\|_{L^{2}\left(\mu_{k}\right)} .
\end{aligned}
$$

Then, by (2.6) we deduce

$$
\left|F_{\eta, g}^{*}(y)\right| \leq\|g\|_{L^{2}\left(\mu_{k}\right)}\left(\int_{\mathbf{R}^{d}} \frac{\chi_{h}(z)|m(z)|^{2} \mathrm{~d} \mu_{k}(z)}{\left[\eta+|m(z)|^{2}\right]^{2}}\right)^{1 / 2} .
$$

Using the fact that

$$
\left[\eta+|m(z)|^{2}\right]^{2} \geq 4 \eta|m(z)|^{2}
$$

we obtain the result.

(ii) We write

$$
F_{\eta, g}^{*}(y)=\int_{\mathbf{R}^{d}} e^{-|x|^{2} / 4} e^{|x|^{2} / 4} g(x) Q_{h}(x, y) \mathrm{d} \mu_{k}(x) .
$$

Applying Hölder's inequality, we obtain

$$
\left|F_{\eta, g}^{*}(y)\right|^{2} \leq \int_{\mathbf{R}^{d}}|g(x)|^{2} e^{|x|^{2} / 2}\left|Q_{h}(x, y)\right|^{2} \mathrm{~d} \mu_{k}(x) .
$$

Thus and from Fubini-Tonnelli's theorem, we get

$$
\left\|F_{\eta, g}^{*}\right\|_{L^{2}\left(\mu_{k}\right)}^{2} \leq \int_{\mathbf{R}^{d}}|g(x)|^{2} e^{|x|^{2} / 2}\left\|Q_{h}(x, .)\right\|_{L^{2}\left(\mu_{k}\right)}^{2} \mathrm{~d} \mu_{k}(x) .
$$
The function $z \rightarrow \frac{\chi_{h}(z) \overline{m(z)} E_{k}(-i x, z)}{\eta+|m(z)|^{2}}$ belongs to $L^{1} \cap L^{2}\left(\mu_{k}\right)$, then by Theorem
2.1 (ii),

$$
Q_{h}(x, y)=\mathscr{F}_{k}^{-1}\left(\frac{\chi_{h}(z) \overline{m(z)} E_{k}(-i x, z)}{\eta+|m(z)|^{2}}\right)(y) .
$$

Thus, by Theorem 2.1 (iii) we deduce that

$$
\left\|Q_{h}(x, .)\right\|_{L^{2}\left(\mu_{k}\right)}^{2}=\int_{\mathbf{R}^{d}}\left|\mathscr{F}_{k}\left(Q_{h}(x, .)\right)(z)\right|^{2} \mathrm{~d} \mu_{k}(z) \leq \int_{\mathbf{R}^{d}} \frac{\chi_{h}(z)|m(z)|^{2} \mathrm{~d} \mu_{k}(z)}{\left[\eta+|m(z)|^{2}\right]^{2}} .
$$


Then using the inequality (3.4), we obtain

$$
\left\|Q_{h}(x, .)\right\|_{L^{2}\left(\mu_{k}\right)} \leq \frac{1}{2 \sqrt{\eta}}\left(\int_{\mathbf{R}^{d}} \chi_{h}(z) \mathrm{d} \mu_{k}(z)\right)^{1 / 2} .
$$

From this inequality and (3.5) we deduce the result.

COROllary 3.4. Let $\eta>0$. For every $g \in L^{2}\left(\mu_{k}\right)$, we have

(i) $F_{\eta, g}^{*}(y)=\int_{\mathbf{R}^{d}} E_{k}(i y, z) \frac{\chi_{h}(z) \overline{m(z)} \mathscr{F}_{k}(g)(z)}{\eta+|m(z)|^{2}} \mathrm{~d} \mu_{k}(z)$.

(ii) $\mathscr{F}_{k}\left(F_{\eta, g}^{*}\right)(z)=\frac{\chi_{h}(z) \overline{m(z)} \mathscr{\mathscr { F }}_{k}(g)(z)}{\eta+|m(z)|^{2}}$.

(iii) $\left\|F_{\eta, g}^{*}\right\|_{H_{h}} \leq \frac{1}{2 \sqrt{\eta}}\|g\|_{L^{2}\left(\mu_{k}\right)}$.

Proof. (i) follows from (3.2) by using Theorem 2.1 (iii) and (2.6).

(ii) The function $z \rightarrow \frac{\chi_{h}(z) \overline{m(z)} \mathscr{F}_{k}(g)(z)}{\eta+|m(z)|^{2}}$ belongs to $L^{1} \cap L^{2}\left(\mu_{k}\right)$. Then by

$$
F_{\eta, g}^{*}(y)=\mathscr{F}_{k}^{-1}\left(\frac{\chi_{h}(z) \overline{m(z)}_{\mathscr{F}_{k}}(g)(z)}{\eta+|m(z)|^{2}}\right)(y) .
$$

Thus, by Theorem 2.1 (iii), we obtain (ii).

(iii) By relation (ii) we have

$$
\left\|F_{\eta, g}^{*}\right\|_{H_{h}}^{2}=\int_{\mathbf{R}^{d}} \frac{\left|\mathscr{F}_{k}\left(f_{\eta, g}^{*}\right)(z)\right|^{2}}{\chi_{h}(z)} \mathrm{d} \mu_{k}(z)=\int_{\mathbf{R}^{d}} \frac{|m(z)|^{2}\left|\mathscr{F}_{k}(g)(z)\right|^{2}}{\left[\eta+|m(z)|^{2}\right]^{2}} \mathrm{~d} \mu_{k}(z) .
$$

Using the inequality (3.4), we obtain

$$
\left\|F_{\eta, g}^{*}\right\|_{H_{h}}^{2} \leq \frac{1}{4 \eta} \int_{\mathbf{R}^{d}}\left|\mathscr{F}_{k}(g)(z)\right|^{2} \mathrm{~d} \mu_{k}(z)=\frac{1}{4 \eta}\|g\|_{L^{2}\left(\mu_{k}\right)}^{2},
$$

which ends the proof.

TheOREM 3.5. Let $\eta>0$. For every $g \in L^{2}\left(\mu_{k}\right)$, we have

(i) $T_{k, m} F_{\eta, g}^{*}(y)=\int_{\mathbf{R}^{d}} E_{k}(i y, z) \frac{\chi_{h}(z)|m(z)|^{2} \mathscr{F}_{k}(g)(z)}{\eta+|m(z)|^{2}} \mathrm{~d} \mu_{k}(z)$.

(ii) $\mathscr{F}_{k}\left(T_{k, m} F_{\eta, g}^{*}\right)(z)=\frac{\chi_{h}(z)|m(z)|^{2} \mathscr{\mathscr { F }}_{k}(g)(z)}{\eta+|m(z)|^{2}}$.

(iii) $T_{k, m} F_{\eta, g}^{*}(y)=F_{\eta, T_{k, m} g}^{*}(y)$.

(iv) $\lim _{\eta \rightarrow 0^{+}}\left\|T_{k, m} F_{\eta, g}^{*}-g\right\|_{L^{2}\left(\mu_{k}\right)}=0$.

Proof. From (2.3) and Corollary 3.4 (ii), we have

$$
T_{k, m} F_{\eta, g}^{*}(y)=\mathscr{F}_{k}^{-1}\left(\frac{\chi_{h}(z)|m(z)|^{2} \mathscr{\mathscr { F }}_{k}(g)(z)}{\eta+|m(z)|^{2}}\right)(y) .
$$


The function $z \rightarrow \frac{\chi_{h}(z)|m(z)|^{2} \mathscr{F}_{k}(g)(z)}{\eta+|m(z)|^{2}}$ belongs to $L^{1} \cap L^{2}\left(\mu_{k}\right)$. Then by Theorem 2.1 (ii), we obtain (i), and by Theorem 2.1 (iii) we obtain (ii).

(iii) follows from (i) and Corollary 3.4 (i).

(iv) From (ii) we have

$$
\widetilde{\mathscr{F}}_{k}\left(T_{k, m} F_{\eta, g}^{*}-g\right)(z)=\frac{-\eta \widetilde{F}_{k}(g)(z)}{\eta+\chi_{h}(z)|m(z)|^{2}} .
$$

Thus,

$$
\left\|T_{k, m} F_{\eta, g}^{*}-g\right\|_{L^{2}\left(\mu_{k}\right)}^{2}=\int_{\mathbf{R}^{d}} \frac{\eta^{2}\left|\mathscr{F}_{k}(g)(z)\right|^{2}}{\left[\eta+\chi_{h}(z)|m(z)|^{2}\right]^{2}} \mathrm{~d} \mu_{k}(z) .
$$

Using the dominated convergence theorem and the fact that

we deduce (iv).

$$
\frac{\eta^{2}\left|\mathscr{F}_{k}(g)(z)\right|^{2}}{\left[\eta+\chi_{h}(z)|m(z)|^{2}\right]^{2}} \leq\left|\mathscr{F}_{k}(g)(z)\right|^{2}
$$

THEOREM 3.6. Let $\eta>0$. For every $f \in H_{h}$, we have

(i) $\lim _{\eta \rightarrow 0^{+}}\left\|F_{\eta, T_{k, m} f}^{*}-f\right\|_{L^{\infty}\left(\mu_{k}\right)}=0$.

(ii) $\lim _{\eta \rightarrow 0^{+}}\left\|F_{\eta, T_{k, m}}^{*}-f\right\|_{H_{h}}=0$.

Proof. (i) From (2.2), the function $\mathscr{F}_{k}(f) \in L^{1} \cap L^{2}\left(\mu_{k}\right)$. Then by Corollary 3.4 (i) and Theorem 2.1 (ii),

$$
F_{\eta, T_{k, m} f}^{*}(y)-f(y)=\int_{\mathbf{R}^{d}} \frac{-\eta \mathscr{F}_{k}(f)(z)}{\eta+\chi_{h}(z)|m(z)|^{2}} E_{k}(i y, z) \mathrm{d} \mu_{k}(z) .
$$

So

$$
\left\|F_{\eta, T_{k, m} f}^{*}-f\right\|_{L^{\infty}\left(\mu_{k}\right)} \leq \int_{\mathbf{R}^{d}} \frac{\eta\left|\mathscr{F}_{k}(f)(z)\right|}{\eta+\chi_{h}(z)|m(z)|^{2}} \mathrm{~d} \mu_{k}(z) .
$$

Again, by dominated convergence theorem and the fact that

$$
\frac{\eta\left|\mathscr{F}_{k}(f)(z)\right|}{\eta+\chi_{h}(z)|m(z)|^{2}} \leq\left|\mathscr{F}_{k}(f)(z)\right|
$$

we deduce (i).

(ii) From (3.7) we have

$$
\mathscr{F}_{k}\left(F_{\eta, T_{k, m} f}^{*}-f\right)(z)=\frac{-\eta \mathscr{F}_{k}(f)(z)}{\eta+\chi_{h}(z)|m(z)|^{2}} .
$$

Consequently,

$$
\left\|F_{\eta, T_{k, m} f}^{*}-f\right\|_{H_{h}}^{2}=\int_{\mathbf{R}^{d}} \frac{\eta^{2}\left|\mathscr{F}_{k}(f)(z)\right|^{2}}{\chi_{h}(z)\left[\eta+\chi_{h}(z)|m(z)|^{2}\right]^{2}} \mathrm{~d} \mu_{k}(z) .
$$


Using the dominated convergence theorem and the fact that

$$
\frac{\eta^{2}\left|\mathscr{F}_{k}(f)(z)\right|^{2}}{\chi_{h}(z)\left[\eta+\chi_{h}(z)|m(z)|^{2}\right]^{2}} \leq \frac{\left|\mathscr{F}_{k}(f)(z)\right|^{2}}{\chi_{h}(z)},
$$

we deduce (ii).

Remark 3.7. $(\eta=0)$. Let $m \in L^{\infty}\left(\mu_{k}\right)$ with $m \neq 0$; and let $g \in L^{2}\left(\mu_{k}\right)$.

(i) From (3.3) we have

$$
\left|F_{0, g}^{*}(y)\right| \leq\left(\int_{\mathbf{R}^{d}} \frac{\chi_{h}(z)}{|m(z)|^{2}} \mathrm{~d} \mu_{k}(z)\right)^{1 / 2}\|g\|_{L^{2}\left(\mu_{k}\right)} .
$$

If we take $g=T_{k, m} f$ when $f \in H_{h}$, then by Theorem 3.6 (i), we get

$$
|f(y)| \leq\left(\int_{\mathbf{R}^{d}} \frac{\chi_{h}(z)}{|m(z)|^{2}} \mathrm{~d} \mu_{k}(z)\right)^{1 / 2}\left\|T_{k, m} f\right\|_{L^{2}\left(\mu_{k}\right)} .
$$

(ii) From (3.6) we have

$$
\left\|Q_{h}(x, .)\right\|_{L^{2}\left(\mu_{k}\right)}^{2} \leq \int_{\mathbf{R}^{d}} \frac{\chi_{h}(z)}{|m(z)|^{2}} \mathrm{~d} \mu_{k}(z) .
$$

Then by (3.5) we obtain

$$
\left\|F_{0, g}^{*}\right\|_{L^{2}\left(\mu_{k}\right)} \leq\left(\int_{\mathbf{R}^{d}} \frac{\chi_{h}(z)}{|m(z)|^{2}} \mathrm{~d} \mu_{k}(z)\right)^{1 / 2}\left(\int_{\mathbf{R}^{d}}|g(x)|^{2} e^{|x|^{2} / 2} \mathrm{~d} \mu_{k}(x)\right)^{1 / 2} .
$$

By Theorem 3.5 (iii) and (iv), we deduce that

$$
\|g\|_{L^{2}\left(\mu_{k}\right)} \leq\left(\int_{\mathbf{R}^{d}} \frac{\chi_{h}(z)}{|m(z)|^{2}} \mathrm{~d} \mu_{k}(z)\right)^{1 / 2}\left(\int_{\mathbf{R}^{d}}\left|T_{k, m} g(x)\right|^{2} e^{|x|^{2} / 2} \mathrm{~d} \mu_{k}(x)\right)^{1 / 2} .
$$

\section{REFERENCES}

[ 1 ] N. Aronszajn, Theory of reproducing kernels, Trans. Amer. Math. Soc. 68 (1948), 337-404.

[2] C. F. DunkL, Integral kernels with reflection group invariance, Canad. J. Math. 43 (1991), 1213-1227.

[ 3 ] C. F. DunkL, Hankel transforms associated to finite reflection groups, Contemp. Math. 138 (1992), 123-138.

[ 4 ] M. F. E. DE JeU, The Dunkl transform, Invent. Math. 113 (1993), 147-162.

[5] G. S. Kimeldorf And G. Wahba, Some results on Tchebycheffian spline functions, J. Math. Anal. Appl. 33 (1971), 82-95.

[6] T. Matsuura, S. Saitoh and D. D. Trong, Inversion formulas in heat conduction multidimensional spaces, J. Inv. Ill-posed Problems 13 (2005), 479-493.

[7] T. MatsuUra and S. Saitoh, Analytical and numerical inversion formulas in the Gaussian convolution by using the Paley-Wiener spaces, Appl. Anal. 85 (2006), 901-915.

[8] E. M. OpdaM, Dunkl operators, Bessel functions and the discriminant of a finite Coxeter group, Compositio Math. 85 (1993), 333-373. 
[9] M. Rösler AND M. Voit, Markov processes related with Dunkl operators, Adv. Appl. Math. 21 (1998), 575-643.

[10] S. SAIтоH, Hilbert spaces induced by Hilbert space valued functions, Proc. Amer. Math. Soc. 89 (1983), 74-78

[11] S. SAItOH, The Weierstrass transform and an isometry in the heat equation, Appl. Anal. 16 (1983), 1-6.

[12] S. Saitoh, Approximate real inversion formulas of the Gaussian convolution, Appl. Anal. 83 (2004), 727-733.

[13] S. SAITOH, Best approximation, Tikhonov regularization and reproducing kernels, Kodai Math. J. 28 (2005), 359-367.

[14] F. Soltani, Inversion formulas in the Dunkl-type heat conduction on $\mathbf{R}^{d}$, Appl. Anal. 84 (2005), 541-553.

[15] F. Soltani, Best approximation formulas for the Dunkl $L^{2}$-multiplier operators on $\mathbf{R}^{d}$, Rocky Mountain J. Math. 42 (2012), 305-328.

[16] F. Soltani, Multiplier operators and extremal functions related to the dual Dunkl-Sonine operator, Acta Math. Sci. 33B (2013), 430-442.

[17] F. Soltani, Inversion formulas for the Dunkl-type Segal-Bargmann transform, Int. Trans. Spec. Funct. 26 (2015), 325-339.

[18] F. Soltani and A. Nemri, Analytical and numerical approximation formulas for the Fourier multiplier operators, Complex Anal. Oper. Theory 9 (2015), 121-138.

[19] M. Yamada, T. MatsuURa AND S. SAITOH, Representations of inverse functions by the integral transform with the sign kernel, Frac. Calc. Appl. Anal. 2 (2007), 161-168.

Fethi Soltani

Department of Mathematics

FaCUlty of SCIENCE

JAZAN UNIVERSITY

P.O. Box 277

JAZAN 45142

SAUdi ARABIA

E-mail: fethisoltani10@yahoo.com 